\title{
Variational Formulation of Rate- and State-Dependent Friction Problems
}

Elias Pipping, Oliver Sander, and

Ralf Kornhuber

Bericht Nr. 359

März 2013

Key words: Continuum mechanics, frictional contact problems, gradient flows, finite elements

AMS Subject Classifications: 65N55, 74F99

Institut für Geometrie und Praktische Mathematik RWTH Aachen

Templergraben 55, D-52056 Aachen (Germany)

This work was partially supported by the Helmholtz graduate research school GEoSim and the DFG Research Center MATHEON. 


\title{
VARIATIONAL FORMULATION OF RATE- AND STATE-DEPENDENT FRICTION PROBLEMS
}

\author{
ELIAS PIPPING, OLIVER SANDER, AND RALF KORNHUBER
}

\begin{abstract}
We propose a variational formulation of rate- and state-dependent models for the dynamic sliding of a linearly elastic block on a rigid surface in terms of two coupled variational inequalities. Classical Dieterich-Ruina models are covered as special cases. We show existence and uniqueness of solutions for the two spatial subproblems arising from time discretisation. Existence of solutions to the coupled spatial problems is established for Dieterich's state equation through a fixed point argument. We conclude with some numerical experiments that suggest mesh independent convergence of the underlying fixed point iteration, and illustrate quasiperiodic occurrence of stick/slip events.
\end{abstract}

\section{INTRODUCTION}

The Dieterich-Ruina model of rate- and state-dependent friction (RSF) 28 has become a standard for frictional behaviour of solids, in particular in the earth sciences 4, 20, 27. It is motivated by so-called velocity stepping tests, in which a block is slid along a foundation and subjected to abrupt changes in sliding velocity (see Figure 1.1). The evolution of the coefficient of friction in such tests reveals two effects: A direct increase/decrease that counteracts the increasing/decreasing sliding velocity, and a relaxation effect, similar to the behaviour of viscoelastic solids.

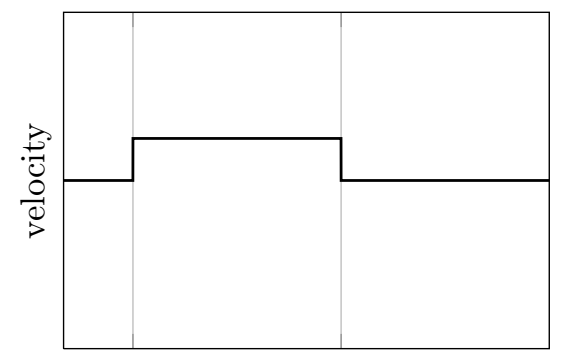

displacement

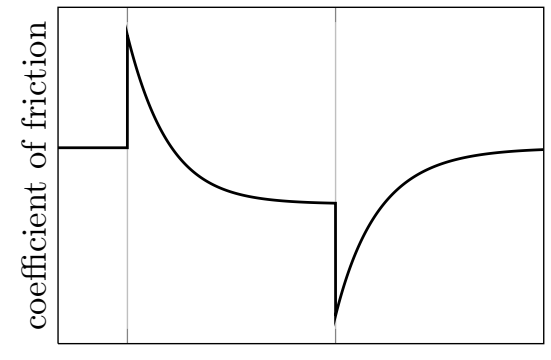

displacement

Figure 1.1. An idealised velocity stepping test

In RSF models, the sliding velocity is often called slip rate. The direct increase/decrease of the coefficient of friction $\mu$ is accounted for through a slip rate-dependence of $\mu$, and the relaxation effect is captured by an additional state variable. While direct slip rate-dependence gives rise to intrinsic instability of stick/slip events, statedependence of $\mu$ has a smoothing effect on the evolution.

Key words and phrases. Continuum mechanics, frictional contact problems, gradient flows, finite elements.

This work was partially supported by the Helmholtz graduate research school GEOSim and the DFG Research Center MAThEon. 
RSF models are inherently coupled, since the evolution of the state variable depends on the slip rate through a suitable equation of state, usually a pointwise ordinary differential equation, and the slip rate in turn depends on the state through a continuum mechanical problem involving the rate- and state-dependent friction coefficient.

In spite of widespread practical applications of RSF, the mathematical properties of this model class have hardly been studied. The most thoroughly investigated setup appears to be the spring-block slider with a single degree of freedom [17, 21, 23, 26. Also, the stability of sliding between two elastic half-spaces has been analysed in 24 .

In this paper, we consider dynamic sliding of a linearly elastic body on a rigid surface. Our model involves Newton's second law together with subdifferential inclusions for friction and state evolution. The variational formulation of this general approach amounts to two variational inequalities that describe the evolution of the slip rate and state, respectively. Special cases include Tresca friction [9, 18, and natural extensions of the classical Dieterich-Ruina model [28]. These extensions include non-smooth evolution of state as well as vanishing velocities, which have been treated by means of regularisation in previous simulations [5, 7]. The timedependent variational inequalities for slip rate and state are discretised in time with the classical Newmark scheme and the backward Euler method, respectively. As a result, two coupled convex minimisation problems have to be solved in each time step. Similar spatial problems are obtained from other implicit time discretisations. We show existence and uniqueness of solutions for each of these subproblems, so that a corresponding fixed point iteration is well defined. Existence of a fixed point is established in the special case of the Dieterich-Ruina model with Dieterich's state equation. We emphasise that corresponding variants of our theoretical results readily extend to a quasistatic variant of the model.

In our numerical experiments, we use piecewise linear and piecewise constant finite elements for the approximation of velocity and state, respectively. Both for Dieterich's and Ruina's state equation, our numerical computations suggest meshindependent convergence rates of a discrete version of the fixed point iteration mentioned above. The resulting approximate displacements and velocities eventually enter a regime of quasi-periodic slip events as expected. For Dieterich's law, we observe grid convergence for fixed spatial mesh and high temporal resolution, while Ruina's law appears to require even smaller time step sizes. More efficient discretisation schemes are the subject of current research.

\section{Rate- And State-Dependent Friction}

2.1. Variational Rate- and State-Dependent Friction. We consider dynamic sliding of a linearly elastic body on a rigid surface. The body shall be represented by a bounded domain $\Omega$ in $\mathbb{R}^{d}$ with Lipschitz boundary. Here, $d$ stands for the spatial dimension. We assume the boundary of $\Omega$ to consist of three subsets $\Gamma_{D}$, $\Gamma_{N}$, and $\Gamma_{F}$ with disjoint relative interiors (Figure 2.1). The letter $n$ is used for the unit outer normal vector of $\Omega$ wherever it is defined.

Suppose that a body force $f$ acts on all of $\Omega$ and a surface force $f_{N}$ acts on the Neumann boundary section $\Gamma_{N}$. We write $u(x, t)$ for the displacement field, which we assume to be prescribed on the Dirichlet boundary section $\Gamma_{D}$. On the remaining section $\Gamma_{F}$ we require the tangential displacement to obey a friction law to be described below. We also assume bilateral contact on $\Gamma_{F}$, i.e. no displacement in the normal direction. This implies that we need not distinguish between the displacement $u$ and its tangential projection $u_{t}$ on $\Gamma_{F}$. 


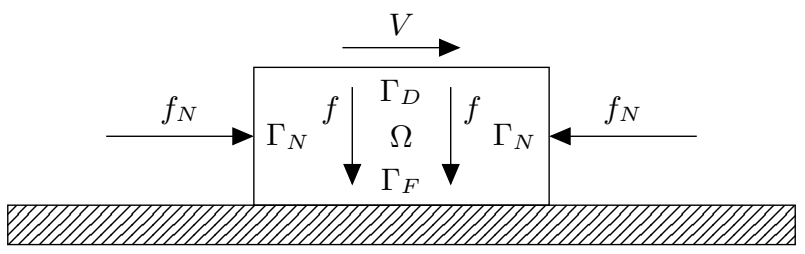

Figure 2.1. A model slider

We impose a rate- and state-dependent friction law of the form

$$
-\sigma_{t} \in \partial_{\dot{u}} \phi(\dot{u}, \alpha) \text {, }
$$

where $\partial_{\dot{u}} \phi(\dot{u}, \alpha)$ denotes the subdifferential of a convex function $\phi(\cdot, \alpha)$ [25]. Here, we wrote $\sigma_{t}:=\boldsymbol{\sigma} n-(\boldsymbol{\sigma} n \cdot n) n$ for the tangential component of the stress field on the boundary, defined through the stress tensor $\sigma$. The evolution of the solutiondependent state variable $\alpha$ is given by

$$
-\dot{\alpha} \in \partial_{\alpha} \psi(\alpha,|\dot{u}|)
$$

with a second convex function $\psi(\cdot,|\dot{u}|)$.

In summary, we consider the following abstract problem of RSF.

Problem. Find $u: \Omega \times[0, T] \rightarrow \mathbb{R}^{d}$ and $\alpha: \Gamma_{F} \times[0, T] \rightarrow \mathbb{R}$ such that

$$
\begin{aligned}
\boldsymbol{\sigma}(u) & =\mathcal{C} \varepsilon(u) & & \text { in } \Omega & & \text { (linear elasticity) } \\
\operatorname{Div} \boldsymbol{\sigma}(u)+f & =\rho \ddot{u} & & \text { in } \Omega & & \text { (balance of momentum) }
\end{aligned}
$$

with boundary conditions

$$
\begin{aligned}
& u=0 \quad \text { on } \Gamma_{D} \\
& \boldsymbol{\sigma}(u) n=f_{N} \quad \text { on } \Gamma_{N} \\
& u_{n}=0 \quad \text { on } \Gamma_{F} \\
& -\sigma_{t} \in \partial_{\dot{u}} \phi(\dot{u}, \alpha) \quad \text { on } \Gamma_{F} \quad \text { (friction law) }
\end{aligned}
$$

and such that $\alpha$ satisfies

$$
-\dot{\alpha} \in \partial_{\alpha} \psi(\alpha,|\dot{u}|) \quad \text { on } \Gamma_{F} \quad \text { (state evolution) }
$$

for all $t \in[0, T]$, where $\rho>0$ is the constant material density, $\mathcal{C}$ is the tensor of elasticity, and $\varepsilon$ is the linearised strain tensor. In addition, we impose initial conditions on the displacement $u$, velocity $\dot{u}$, and state $\alpha$.

Remark. We have assumed homogeneous Dirichlet boundary conditions, i.e., $u=0$ on $\Gamma_{D}$. This assumption serves mainly to simplify the presentation; in Section 6 . we consider a numerical experiment with inhomogeneous Dirichlet boundary conditions.

Assuming that the state $\alpha$ is known, this problem can be written as a variational inequality for $u \in H^{1}((0, T), H) \cap H^{2}\left((0, T), H^{*}\right)$ with

$$
H:=\left\{w \in H^{1}(\Omega)^{d}:\left.w\right|_{\Gamma_{D}}=0,\left.w_{n}\right|_{\Gamma_{F}}=0\right\} .
$$

Consider the balance of momentum equation (2.2). After testing with $v-\dot{u}(t)$, $v \in H$, and using (2.1) as well as the boundary conditions for $u$ and $u_{n}$, we obtain

$$
\langle\rho \ddot{u}, v-\dot{u}\rangle+a(u, v-\dot{u})=\ell(v-\dot{u})+\int_{\Gamma_{F}} \sigma_{t}(u) \cdot(v-\dot{u}) \quad \forall v \in H,
$$

where $\langle\cdot, \cdot\rangle$ denotes the dual pairing of $H$ and $H^{*}$. Also, we have set

$$
a(v, w):=\int_{\Omega} \mathcal{C} \varepsilon(v): \varepsilon(w), \text { as well as } \ell(v):=\int_{\Omega} f \cdot v+\int_{\Gamma_{N}} f_{N} \cdot v .
$$


We now recall that 2.3 can be equivalently written as

$$
\sigma_{t}(x) \cdot[v-\dot{u}(x)]+\phi(v, \alpha) \geq \phi(\dot{u}(x), \alpha) \quad \forall v \in \mathbb{R}^{d}
$$

at any point $x \in \Gamma_{F}$. Testing 2.6 with traces of functions from $H$ yields the weaker form

with

$$
\Phi(v, \alpha) \geq \Phi(\dot{u}, \alpha)-\sigma_{t}(v-\dot{u}) \quad \forall v \in H
$$

$$
\Phi(v, \alpha):=\int_{\Gamma_{F}} \phi(v, \alpha) .
$$

Here, we assume $\phi$ to be chosen such that $\Phi$ is well-defined. Combined with (2.5) this leads to a variational formulation of the elastic problem with given state $\alpha$.

Problem (R). For given $\alpha$, find $u \in H^{1}((0, T), H) \cap H^{2}\left((0, T), H^{*}\right)$ such that for almost every $t \in[0, T]$, we have

$$
\langle\rho \ddot{u}, v-\dot{u}\rangle+a(u, v-\dot{u})+\Phi(v, \alpha) \geq \Phi(\dot{u}, \alpha)+\ell(v-\dot{u}) \quad \forall v \in H .
$$

Analogously, we can formulate a variational problem for the state variable $\alpha$ under the assumption that $|\dot{u}|$ is known. To that end, we test 2.4 with functions $\beta \in$ $L^{2}\left(\Gamma_{F}\right)$ and define a functional through

$$
\Psi(\beta, V)=\int_{\Gamma_{F}} \psi(\beta, V),
$$

with $\psi$ such that $\Psi$ is well-defined, to obtain the following variational formulation of $(2.4)$.

Problem (S). For given $|\dot{u}|$, find $\alpha \in H^{1}\left((0, T), L^{2}\left(\Gamma_{F}\right)\right)$ such that for almost every $t \in[0, T]$, we have

$$
(\dot{\alpha}, \beta-\alpha)_{L^{2}\left(\Gamma_{F}\right)}+\Psi(\beta,|\dot{u}|) \geq \Psi(\alpha,|\dot{u}|) \quad \forall \beta \in L^{2}(\Gamma) .
$$

Here, $(\cdot, \cdot)_{L^{2}\left(\Gamma_{F}\right)}$ denotes the scalar product in $L^{2}\left(\Gamma_{F}\right)$. The variational formulation of the coupled rate- and state-dependent friction problem finally reads

Problem (RSF). Find $u \in H^{1}((0, T), H) \cap H^{2}\left((0, T), H^{*}\right)$ and $\alpha \in H^{1}\left((0, T), L^{2}\left(\Gamma_{F}\right)\right)$ such that for almost every $t \in[0, T]$, we have

$$
\begin{array}{rlrl}
\langle\rho \ddot{u}, v-\dot{u}\rangle+a(u, v-\dot{u})+\Phi(v, \alpha) \geq \Phi(\dot{u}, \alpha)+\ell(v-\dot{u}) & & \forall v \in H, \\
(\dot{\alpha}, \beta-\alpha)_{L^{2}\left(\Gamma_{F}\right)}+\Psi(\beta,|\dot{u}|) \geq \Psi(\alpha,|\dot{u}|) & \forall \beta \in L^{2}(\Gamma) .
\end{array}
$$

2.2. Tresca Friction. Coulomb friction [9, 18, postulates that tangential stress $\sigma_{t}$ and velocity $\dot{u}$ are related according to

$$
-\sigma_{t}=\mu\left|\sigma_{n}\right| \frac{\dot{u}}{|\dot{u}|} \quad \text { if } \dot{u} \neq 0 \quad \text { and } \quad\left|\sigma_{t}\right| \leq \mu\left|\sigma_{n}\right| \quad \text { if } \dot{u}=0,
$$

with a given friction coefficient $\mu \geq 0$. Tresca friction is obtained by replacing the solution-dependent normal stress $\sigma_{n}<0$ by a given parameter $\bar{\sigma}_{n}$. Since the subdifferential of the Euclidean norm $|\cdot|$ is given by

$$
\partial|\cdot|(\dot{u})= \begin{cases}\{\dot{u} /|\dot{u}|\} & \text { if } \dot{u} \neq 0 \\ \left\{x \in \mathbb{R}^{d}:|x| \leq 1\right\} & \text { if } \dot{u}=0\end{cases}
$$

the friction law (2.7) with $\sigma_{n}=\bar{\sigma}_{n}$ can be equivalently written in the form (2.3). The convex function $\phi$ is then given by

$$
\phi(\dot{u})=\mu\left|\bar{\sigma}_{n}\right||\dot{u}| .
$$

A state-dependent extension of classical Tresca friction 2.8 can be introduced by replacing $\phi(\dot{u})$ with $\phi(\dot{u}, \alpha)=\mu(\alpha)\left|\bar{\sigma}_{n}\right||\dot{u}|$, involving a state-dependent friction coefficient $\mu(\alpha)$ and a state evolution law of the form (2.4). 
2.3. The Dieterich-Ruina Model. The Dieterich-Ruina model of RSF in its most common form goes back to [28] and consists of two parts: An equation that relates the coefficient of friction $\mu$ to the slip rate $V:=|\dot{u}|$ and a state $\theta$, as well as a state evolution equation. The former is most commonly stated as [1, 3, 10, 19, 22, 29 ,

$$
\mu:=\frac{\left|\sigma_{t}\right|}{\left|\sigma_{n}\right|}=\mu_{0}+a \log \left(\frac{V}{V_{0}}\right)+b \log \left(\frac{V_{0} \theta}{L}\right)
$$

with positive parameters $\mu_{0}, a, b, V_{0}$, and $L \in \mathbb{R}$.

For the second equation, multiple proposals have been made. The two most popular laws are given by

$$
\dot{\theta}=1-\frac{V}{L} \theta \quad \text { (Dieterich's law) }
$$

and

$$
\dot{\theta}=-\frac{V}{L} \theta \log \left(\frac{V}{L} \theta\right) \quad \text { (Ruina's law). }
$$

Both can be used to describe some phenomena but not others [1, 19].

As a common feature, both of these state equations provide increasing state $\theta$ for small slip rate $V$ and vice versa. A variety of state equations with this characteristic property might be useful. Consider, e.g., the most simple but non-smooth law

$$
\dot{\theta}=\left\{\begin{array}{ll}
+V & \text { if } \theta V<1 \\
-V & \text { if } \theta V>1
\end{array} \text { and } \quad|\dot{\theta}| \leq V \quad \text { if } \theta V=1\right.
$$

We replace the solution-dependent normal stress $\sigma_{n}$ in $(2.9)$ with a parameter $\bar{\sigma}_{n}$ (as is done in Tresca friction) and assume collinearity of velocity and stress, i.e.,

$$
-\left|\sigma_{t}\right| \dot{u}=|\dot{u}| \sigma_{t}
$$

Then the Dieterich-Ruina model becomes a special case of the framework set forth in Section 2.1.

To show that, we first relate $(2.9)$ and $(2.3)$. It is obvious that $(2.9)$ is not meaningful for very low velocities $V$, since for fixed $\theta$ and $V \rightarrow 0$ the right-hand side tends to $-\infty$, whereas the left-hand side remains non-negative. This problem has been circumvented in the literature by means of regularisation [5, 7]; in what follows, we follow a variational approach.

To give a precise bound from which on velocities become inadmissible to (2.9), we set

so that $(2.9)$ becomes

$$
V_{m}(\theta):=V_{0} \exp \left(-\frac{\mu_{0}+b \log \left(\theta V_{0} / L\right)}{a}\right)
$$

$$
\left|\sigma_{t}\right| /\left|\bar{\sigma}_{n}\right|=a \log \left(V / V_{0}\right)-a \log \left(V_{m} / V_{0}\right) .
$$

This formulation makes it clear that we must have $V \geq V_{m}$ for $(2.9)$ to make sense. A straightforward extension of $(2.9)$ to velocities that fall short of $V_{m}$ is given by

$$
\mu= \begin{cases}\mu_{0}+a \log \left(V / V_{0}\right)+b \log \left(V_{0} \theta / L\right) & \text { if } V \geq V_{m}(\theta) \\ 0 & \text { otherwise. }\end{cases}
$$

In conjunction with the collinearity assumption 2.11 , this expression can be reformulated as a subdifferential inclusion of type (2.3). Indeed, for $V \geq V_{m}(\theta)$, we have

where $\varphi$ is given by

$$
\left|\sigma_{t}\right|=a\left|\bar{\sigma}_{n}\right| \log \left(V / V_{m}(\theta)\right)=\frac{\partial \varphi}{\partial V}(V, \theta)
$$

$$
\varphi(V, \theta):=a\left|\bar{\sigma}_{n}\right|\left[V \log \left(V / V_{m}(\theta)\right)-V+V_{m}(\theta)\right], \quad V \geq V_{m}(\theta) .
$$


This function is convex and non-decreasing in $V$, a property that is shared by the extension

$$
\varphi(V, \theta)= \begin{cases}a\left|\bar{\sigma}_{n}\right|\left[V \log \left(V / V_{m}(\theta)\right)-V+V_{m}(\theta)\right] & \text { if } V \geq V_{m}(\theta) \\ 0 & \text { otherwise }\end{cases}
$$

corresponding to 2.12 , too, since $\varphi(\cdot, \theta)$ and $\partial \varphi / \partial V(\cdot, \theta)$ vanish at $V_{m}(\theta)$. If we now define

$$
\phi(\cdot, \theta):=\varphi(|\cdot|, \theta)
$$

we obtain

$$
-\sigma_{t}=\frac{\partial \varphi}{\partial V}(V, \theta) \frac{\dot{u}}{|\dot{u}|}=\frac{\partial \phi}{\partial \dot{u}}(\dot{u}, \theta)
$$

by virtue of (2.11) and the chain rule, and thus a smooth case of the subdifferential inclusion (2.3) as desired.

It remains to be shown that the evolution of $\theta$ can be written as a subdifferential inclusion of type (2.4). If we set $\alpha:=\log \theta$ and rewrite Dieterich's law in terms of $\alpha$, it becomes

$$
-\dot{\alpha}=\frac{V}{L}-e^{-\alpha}=\frac{d}{d \alpha} \psi_{d}(\alpha, V) \quad \text { with } \psi_{d}(\alpha, V)=\frac{V}{L} \alpha+e^{-\alpha} .
$$

With the same substitution, Ruina's law turns into $\dot{\alpha}=-V / L(\alpha+\log (V / L))$, or

$$
-\dot{\alpha}=\frac{d}{d \alpha} \psi_{r}(\alpha, V) \text { with } \psi_{r}(\alpha, V)=\frac{V}{L}\left(\frac{1}{2} \alpha^{2}+\log \left(\frac{V}{L}\right) \alpha\right) .
$$

For the discontinuous law 2.10 , we set $\alpha:=\theta$ to obtain

$$
-\dot{\alpha} \in \partial_{\alpha} \psi_{d c}(\alpha, V) \quad \text { with } \quad \psi_{d c}(\alpha, V)=|\alpha V-1| .
$$

Since the functions $\psi_{d}(\cdot, V), \psi_{r}(\cdot, V)$, and $\psi_{d c}$ are convex, the corresponding state equations are again special cases of 2.4 .

\section{Time-Discretisation}

As a first step towards the numerical solution of the coupled variational Problem (RSF) stated in Subsection 2.1, we now consider time-discretisations of the Subproblems (R) and (S). For simplicity, we assume the interval $[0, T]$ to be partitioned uniformly into $N$ subintervals $\left[t_{n-1}, t_{n}\right]$, each of length $\tau=T / N$.

To Subproblem (R) we apply the classical Newmark scheme, which we can write as

$$
\begin{aligned}
& \dot{u}_{n}=\dot{u}_{n-1}+\frac{\tau}{2}\left(\ddot{u}_{n-1}+\ddot{u}_{n}\right) \\
& u_{n}=u_{n-1}+\tau \dot{u}_{n-1}+\frac{\tau^{2}}{4}\left(\ddot{u}_{n-1}+\ddot{u}_{n}\right)
\end{aligned}
$$

for the spatial approximations $u_{n}:=u\left(t_{n}\right)$ and $0<n \leq N$. Note that we can also write (3.1) as

$$
\ddot{u}_{n}=\frac{2}{\tau}\left(\dot{u}_{n}-\dot{u}_{n-1}\right)-\ddot{u}_{n-1},
$$

which we can insert into 3.2 to obtain

$$
u_{n}=u_{n-1}+\frac{\tau}{2}\left(\dot{u}_{n}+\dot{u}_{n-1}\right)
$$

It is easy to see that an application of Newmark's method in this form to Subproblem $(\mathrm{R})$ leads to a variational inequality over $H$ where the sole unknown is $\dot{u}_{n}$ :

$$
a_{\tau}\left(\dot{u}_{n}, v-\dot{u}_{n}\right)+\Phi(v, \alpha) \geq \Phi\left(\dot{u}_{n}, \alpha\right)+\ell_{n}\left(v-\dot{u}_{n}\right) \quad \forall v \in H
$$


with

and

$$
a_{\tau}(\cdot, \cdot):=\frac{2}{\tau}(\rho \cdot, \cdot)_{L^{2}(\Omega)}+\frac{\tau}{2} a(\cdot, \cdot)
$$

$$
\ell_{n}(\cdot):=\ell(\cdot)+\left(\rho\left(\frac{2}{\tau} \dot{u}_{n-1}+\ddot{u}_{n-1}\right), \cdot\right)_{L^{2}(\Omega)}-a\left(u_{n-1}+\frac{\tau}{2} \dot{u}_{n-1}, \cdot\right) .
$$

This variational problem for $\dot{u}_{n}$ can also be written as a minimisation problem; the corresponding energy functional $\mathcal{J}(\cdot, \alpha)$ is given by

$$
\mathcal{J}(v, \alpha)=\frac{1}{2} a_{\tau}(v, v)+\Phi(v, \alpha)-\ell_{n}(v) .
$$

The Newmark time-discretisation of Subproblem $(\mathrm{R})$ thus leads to the following spatial problems.

Problem $\left(\mathrm{R}_{\tau}\right)$. For given state $\alpha$, find $\dot{u}_{n} \in H$ such that

$$
\mathcal{J}\left(\dot{u}_{n}, \alpha\right) \leq \mathcal{J}(v, \alpha) \quad \forall v \in H .
$$

The displacement $u_{n}$ can then be computed from $\dot{u}_{n}$ using (3.4).

Next, we apply the backward Euler scheme to the $L^{2}$-gradient flow Subproblem (S). The spatial approximations $\alpha_{n}:=\alpha\left(t_{n}\right), 0<n \leq N$, then satisfy the variational inequality

$$
\left(\alpha_{n}, \beta-\alpha_{n}\right)_{L^{2}\left(\Gamma_{F}\right)}+\tau \Psi(\beta,|\dot{u}|) \geq \tau \Psi\left(\alpha_{n},|\dot{u}|\right)+\left(\alpha_{n-1}, \beta-\alpha_{n}\right)_{L^{2}\left(\Gamma_{F}\right)}
$$

for all $\beta \in L^{2}\left(\Gamma_{F}\right)$. Since 3.5 can be equivalently written as a minimisation problem for the convex energy functional $\mathcal{E}(\cdot,|\dot{u}|)$ given by

$$
\mathcal{E}(\beta,|\dot{u}|)=\frac{1}{2}(\beta, \beta)_{L^{2}\left(\Gamma_{F}\right)}+\tau \Psi(\beta,|\dot{u}|)-\left(\alpha_{n-1}, \beta\right)_{L^{2}\left(\Gamma_{F}\right)},
$$

we obtain the following spatial problem in each time step.

Problem $\left(\mathrm{S}_{\tau}\right)$. For given slip rate $V=|\dot{u}|$, find $\alpha_{n} \in L^{2}\left(\Gamma_{F}\right)$ such that

$$
\mathcal{E}\left(\alpha_{n}, V\right) \leq \mathcal{E}(\beta, V) \quad \forall \beta \in L^{2}\left(\Gamma_{F}\right) .
$$

The spatial problems of the time-discretised coupled Problem (RSF) finally read as follows.

Problem $\left(\mathrm{RSF}_{\tau}\right)$. Find $\dot{u}_{n} \in H$ and $\alpha_{n} \in L^{2}\left(\Gamma_{F}\right)$ such that

$$
\begin{aligned}
\mathcal{J}\left(\dot{u}_{n}, \alpha_{n}\right) \leq \mathcal{J}\left(v, \alpha_{n}\right) \quad \forall v \in H \\
\mathcal{E}\left(\alpha_{n},\left|\dot{u}_{n}\right|\right) \leq \mathcal{E}\left(\beta,\left|\dot{u}_{n}\right|\right) \quad \forall \beta \in L^{2}\left(\Gamma_{F}\right) .
\end{aligned}
$$

4. Existence and Uniqueness of Solutions of the Spatial Subproblems

Existence and uniqueness of solutions of the spatial Subproblems $\left(\mathrm{R}_{\tau}\right)$ and $\left(\mathrm{S}_{\tau}\right)$ will be derived from the following general results on convex minimisation and superposition operators.

Lemma 4.1. Let $\mathcal{V}$ be a Hilbert space. If we assume that $b(\cdot, \cdot)$ is a symmetric, continuous and $\mathcal{V}$-elliptic bilinear form, $j: \mathcal{V} \rightarrow \mathbb{R} \cup\{+\infty\}$ a proper, convex, and lower semicontinuous functional, and $l$ a bounded linear functional on $\mathcal{V}$, then the problem of minimising

$$
v \mapsto \frac{1}{2} b(v, v)+j(v)-l(v)
$$

over $\mathcal{V}$ admits a unique solution.

Proof. See [13, Lemma 4.1].

It is a straightforward consequence of Fatou's Lemma that integral operators preserve lower semicontinuity. 
Lemma 4.2. Assume that $f: \Gamma_{F} \times \mathbb{R}^{d} \rightarrow \mathbb{R}$ is a non-negative function, such that $f(x, \cdot)$ is lower semicontinuous for almost every $x \in \Gamma_{F}$. Then the superposition operator

$$
\int_{\Gamma_{F}} f(x, \cdot) d x: L^{2}\left(\Gamma_{F}\right) \rightarrow \mathbb{R} \cup\{+\infty\}
$$

is lower semicontinuous.

Proof. See [11, Theorem 6.49].

Now we are ready to show existence and uniqueness of a solution of Subproblem $\left(\mathrm{R}_{\tau}\right)$ under the additional assumption that

(A.1) $\mathcal{C}$ is elliptic, i.e. there exists a $c>0$ such that a.e. in $\Omega$, we have

$$
\mathcal{C} \boldsymbol{\tau}: \boldsymbol{\tau} \geq c|\boldsymbol{\tau}|^{2} \text { for every symmetric tensor } \boldsymbol{\tau} .
$$

Proposition 4.3. Assume that (A.1) holds, $u_{n-1}, \dot{u}_{n-1}, \ddot{u}_{n-1} \in H$, and $\ell \in H^{*}$. Then the Subproblem $\left(R_{\tau}\right)$ with $\phi$ corresponding to Tresca friction $(2.8)$ or the Dieterich-Ruina model (2.14) has a unique solution for any given state $\alpha \in L^{2}\left(\Gamma_{F}\right)$.

Proof. Both for Tresca friction $(2.8)$ and the Dieterich-Ruina model $(2.14)$ the superposition operator $\Phi(\cdot, \alpha)$ is convex, because so is $\phi(\cdot, \alpha)$. It is proper, because $\phi(0, \alpha)=0$. Since in both cases $\phi$ is continuous and non-negative, Lemma 4.2 implies that $\Phi(\cdot, \alpha)$ is lower semicontinuous. The bilinear form $a_{\tau}(\cdot, \cdot)$ is symmetric, continuous, and, by assumption (A.1) in conjunction with Korn's second inequality [30, elliptic on $H$. Hence, the claim follows from Lemma 4.1.

We now consider existence and uniqueness for Subproblem $\left(\mathrm{S}_{\tau}\right)$ under the assumption that

(A.2) $\log L \in L^{\infty}\left(\Gamma_{F}\right)$.

Proposition 4.4. Assume $\alpha_{n-1} \in L^{2}\left(\Gamma_{F}\right)$. Then the Subproblem $\left(S_{\tau}\right)$ with $\psi$ corresponding to the state evolution laws of Dieterich (2.15), Ruina (2.16), or (2.17) has a unique solution for any given slip rate $V=|\dot{u}| \in L^{2+} \delta\left(\Gamma_{F}\right)$ with $\delta \geq 0, \delta>0$, or $\delta \geq 0$, respectively.

Proof. For fixed $V=|\dot{u}| \geq 0$, the convexity of the functionals $j:=\tau \Psi(\cdot, V)=$ $\tau \int_{\Gamma_{F}} \psi(\cdot, V)$ follows immediately from the convexity of $\psi(\cdot, V)$. From $\psi_{r}(0, V)=$ $0, \psi_{d}(0, V)=1$, and $\left|\psi_{d c}(0, V)\right| \leq 1$, we conclude that $\Psi$ is proper.

To show that $\Psi$ is lower semicontinuous, we decompose $\psi$ into its linear and nonlinear parts. For $\psi_{d}$ and $\psi_{r}$, this leads to

$$
\begin{array}{lll}
\psi_{d}=\psi_{d, 1}+\psi_{d, 2}, & \psi_{d, 1}: \alpha \mapsto \frac{V}{L} \alpha, & \psi_{d, 2}: \alpha \mapsto e^{-\alpha}, \\
\psi_{r}=\psi_{r, 1}+\psi_{r, 2}, & \psi_{r, 1}: \alpha \mapsto \frac{V}{L} \log (V / L) \alpha, & \psi_{r, 2}: \alpha \mapsto \frac{1}{2} \frac{V}{L} \alpha^{2} .
\end{array}
$$

Since $\psi_{d, 1}$ and $\psi_{r, 1}$ multiply their arguments with functions that lie in $L^{2}$ by assumption (namely $V \in L^{2+\delta}\left(\Gamma_{F}\right)$ and (A.2)), their respective contribution to $\Psi$ is continuous in $\alpha$. A similar observation shows that $\Psi$ is continuous if $\psi=\psi_{d c}$.

From the non-negativity of $\psi_{d, 2}$ and $\psi_{r, 2}$ we conclude that the corresponding integrals are well-defined and that Lemma 4.2 applies.

Because of $\rho>0$, the bilinear form $(\cdot, \cdot)_{L^{2}\left(\Gamma_{F}\right)}$ is symmetric, continuous, and elliptic on $L^{2}\left(\Gamma_{F}\right)$. Since we assumed $\alpha_{n-1} \in L^{2}\left(\Gamma_{F}\right)$, the claim now follows from Lemma 4.1 . 


\section{Existence of solutions for a Coupled Spatial Dieterich-Ruina}

PROBLEM

Since the Dieterich-Ruina model of RSF with Dieterich's or Ruina's state evolution law leads to subproblems that are uniquely solvable, we can define a solution operator for each problem, namely

$$
S: L^{2+\delta}\left(\Gamma_{F}\right) \rightarrow L^{2}\left(\Gamma_{F}\right) \quad \text { corresponding to Subproblem }\left(\mathrm{S}_{\tau}\right)
$$

and

$$
R: L^{2}\left(\Gamma_{F}\right) \rightarrow H \quad \text { corresponding to Subproblem }\left(\mathrm{R}_{\tau}\right),
$$

for any $\delta \geq 0$. In this manner, we map scalar velocities on $\Gamma_{F}$ to states (using $S$ ) and states to velocity fields on $\Omega$ (using $R$ ). Since such velocity fields can be transformed into scalar velocities on $\Gamma_{F}$ through the map

$$
\left|\gamma_{F}\right|: H \rightarrow L^{p}\left(\Gamma_{F}\right), \quad v \mapsto\left(\Gamma_{F} \ni x \mapsto|v(x)|\right),
$$

we can close the circle here. Before we discuss for which $\delta$ this is possible, we consider the consequences. We first compose the two solution operators to obtain

$$
R S:=R \circ S: L^{2+\delta}\left(\Gamma_{F}\right) \rightarrow H .
$$

If $\left|\gamma_{F}\right|$ now maps to $L^{2+\delta}\left(\Gamma_{F}\right)$, we have a self-map $R S \circ\left|\gamma_{F}\right|: H \rightarrow H$. By construction, a fixed point of this map and its corresponding state solve both Subproblem $\left(\mathrm{S}_{\tau}\right)$ and Subproblem $\left(\mathrm{R}_{\tau}\right)$ simultaneously and thus the coupled Problem $\left(\mathrm{RSF}_{\tau}\right)$.

Now back to $\delta$ : Since the trace operator $\gamma: H \rightarrow L^{p}\left(\Gamma_{F}\right)^{d}$ is well-defined and compact for $1 \leq p<p^{*}$, so is $\left|\gamma_{F}\right|$, with $p^{*}=4$ in the three-dimensional case and $p^{*}=\infty$ in two dimensions [6]. Any $\delta<p^{*}-2$ is thus admissible.

In what follows, we make the necessary arrangements for an application of Schauder's theorem which guarantees that $R S \circ\left|\gamma_{F}\right|$ has a fixed point. To that end, we are forced to restrict ourselves to Dieterich's state evolution law for reasons that become clear as we proceed. We also make the assumptions

(A.3) $\bar{\sigma}_{n} \in L^{\infty}\left(\Gamma_{F}\right)$,

(A.4) $V_{0} \in L^{\infty}\left(\Gamma_{F}\right)$ and $\log V_{0} \in L^{\infty}\left(\Gamma_{F}\right)$,

(A.5) $a \in L^{\infty}\left(\Gamma_{F}\right)$,

(A.6) $b \in L^{\infty}\left(\Gamma_{F}\right)$, and

(A.7) $\mu_{0} \in L^{\infty}\left(\Gamma_{F}\right)$.

5.1. Towards boundedness and continuity of $R S$. Since for any two states $\alpha$, $\beta \in L^{2}\left(\Gamma_{F}\right)$ we have

$$
\begin{aligned}
& \Phi(R(\beta), \alpha)-\Phi(R(\alpha), \alpha)+\Phi(R(\alpha), \beta)-\Phi(R(\beta), \beta) \\
& \quad \geq a_{\tau}(R(\alpha)-R(\beta), R(\alpha)-R(\beta)) \\
& \quad \geq C\|R(\alpha)-R(\beta)\|^{2}
\end{aligned}
$$

for a constant $C>0$ by $H$-ellipticity of $a_{\tau}(\cdot, \cdot)$, upper bounds for the term

$$
\begin{aligned}
\Phi(R(\beta), \alpha)-\Phi(R(\alpha), \alpha)+\Phi(R(\alpha), \beta)-\Phi(R(\beta), \beta) \\
=\int_{\Gamma_{F}} \phi(R(\beta), \alpha)-\phi(R(\alpha), \alpha)+\phi(R(\alpha), \beta)-\phi(R(\beta), \beta)
\end{aligned}
$$

also yield upper bounds for the term $\|R(\alpha)-R(\beta)\|^{2}$. By means of such bounds we establish that $R S$ is bounded (i.e. it maps bounded sets into bounded sets) and continuous. 
Proposition 5.1. We have

$$
\begin{aligned}
\phi(U, \alpha)-\phi(V, \alpha)+\phi(V, \beta)-\phi(U, \beta) \\
\quad \leq a\left|\bar{\sigma}_{n}\right|\left[\max (U, V)\left|\log \left(V_{m}(\alpha) / V_{m}(\beta)\right)\right|+\left|V_{m}(\alpha)-V_{m}(\beta)\right|\right]
\end{aligned}
$$

with $\alpha, \beta, U, V \in \mathbb{R}$ and $0 \leq U, V$.

Proof. Since the function $\varphi$ from 2.13 is defined piecewise depending on whether the first argument is smaller than $V_{m}$ or not, and we consider four terms at once, we can distinguish 16 cases, each of which yields an explicit expression for (5.2). Given that 5.2 remains unchanged if we swap $U$ and $V$ simultaneously with $\alpha$ and $\beta$, some cases are analogous to others. For brevity, we write

$$
(*)=[\phi(U, \alpha)-\phi(V, \alpha)+\phi(V, \beta)-\phi(U, \beta)] / a\left|\bar{\sigma}_{n}\right|
$$

and find:

(1) $U \leq V_{m}(\alpha), V \leq V_{m}(\alpha), V \leq V_{m}(\beta), U \leq V_{m}(\beta)$ : Implies $(*)=0$.

(2) $U \leq V_{m}(\alpha), V \leq V_{m}(\alpha), V \leq V_{m}(\beta), U \geq V_{m}(\beta)$ : Implies $(*) \leq 0$.

(3) $U \leq V_{m}(\alpha), V \leq V_{m}(\alpha), V \geq V_{m}(\beta), U \leq V_{m}(\beta)$ : Implies

$$
(*)=V \log \left(V / V_{m}(\beta)\right)-V+V_{m}(\beta) \leq V \log \left(V_{m}(\alpha) / V_{m}(\beta)\right) .
$$

(4) $U \leq V_{m}(\alpha), V \leq V_{m}(\alpha), V \geq V_{m}(\beta), U \geq V_{m}(\beta)$ : Implies

$$
V_{m}(\alpha) \geq V, U \geq V_{m}(\beta)
$$

and thus

$$
\begin{aligned}
(*) & =V \log \left(V / V_{m}(\beta)\right)-U \log \left(U / V_{m}(\beta)\right)+U-V \\
& \leq V \log \left(V_{m}(\alpha) / V_{m}(\beta)\right)+\left|V_{m}(\alpha)-V_{m}(\beta)\right| .
\end{aligned}
$$

(5) $U \leq V_{m}(\alpha), V \geq V_{m}(\alpha), V \leq V_{m}(\beta), U \leq V_{m}(\beta)$ : Analogous to (2)

(6) $U \leq V_{m}(\alpha), V \geq V_{m}(\alpha), V \leq V_{m}(\beta), U \geq V_{m}(\beta)$ : Implies

$$
U=V_{m}(\alpha)=V=V_{m}(\beta)=U
$$

and thus $(*)=0$.

(7) $U \leq V_{m}(\alpha), V \geq V_{m}(\alpha), V \geq V_{m}(\beta), U \leq V_{m}(\beta)$ : Implies

$$
(*)=V \log \left(V_{m}(\alpha) / V_{m}(\beta)\right)+V_{m}(\beta)-V_{m}(\alpha) .
$$

(8) $U \leq V_{m}(\alpha), V \geq V_{m}(\alpha), V \geq V_{m}(\beta), U \geq V_{m}(\beta)$ : Implies

$$
\begin{aligned}
(*) & =V \log \left(V_{m}(\alpha) / V_{m}(\beta)\right)-U \log \left(U / V_{m}(\beta)\right)+U-V_{m}(\alpha) \\
& \leq V \log \left(V_{m}(\alpha) / V_{m}(\beta)\right) .
\end{aligned}
$$

(9) $U \geq V_{m}(\alpha), V \leq V_{m}(\alpha), V \leq V_{m}(\beta), U \leq V_{m}(\beta)$ : Analogous to (3)

(10) $U \geq V_{m}(\alpha), V \leq V_{m}(\alpha), V \leq V_{m}(\beta), U \geq V_{m}(\beta)$ : Analogous to (7).

(11) $U \geq V_{m}(\alpha), V \leq V_{m}(\alpha), V \geq V_{m}(\beta), U \leq V_{m}(\beta)$ : Implies

$$
U=V_{m}(\alpha)=V=V_{m}(\beta)=U
$$

and thus $(*)=0$.

(12) $U \geq V_{m}(\alpha), V \leq V_{m}(\alpha), V \geq V_{m}(\beta), U \geq V_{m}(\beta)$ : Implies

$$
\begin{aligned}
(*) & =U \log \left(V_{m}(\beta) / V_{m}(\alpha)\right)-V \log \left(V_{m}(\beta) / V\right)+V_{m}(\alpha)-V \\
& \leq U \log \left(V_{m}(\beta) / V_{m}(\alpha)\right)-V \log \left(V_{m}(\beta) / V_{m}(\alpha)\right)+V_{m}(\alpha)-V \\
& \leq V_{m}(\alpha)-V \leq V_{m}(\alpha)-V_{m}(\beta) .
\end{aligned}
$$

(13) $U \geq V_{m}(\alpha), V \geq V_{m}(\alpha), V \leq V_{m}(\beta), U \leq V_{m}(\beta)$ : Analogous to (4)

(14) $U \geq V_{m}(\alpha), V \geq V_{m}(\alpha), V \leq V_{m}(\beta), U \geq V_{m}(\beta)$ : Analogous to (8),

(15) $U \geq V_{m}(\alpha), V \geq V_{m}(\alpha), V \geq V_{m}(\beta), U \leq V_{m}(\beta)$ : Analogous to (12). 
(16) $U \geq V_{m}(\alpha), V \geq V_{m}(\alpha), V \geq V_{m}(\beta), U \geq V_{m}(\beta)$ : Implies

$$
(*)=(U-V) \log \left(V_{m}(\beta) / V_{m}(\alpha)\right) .
$$

The claim now follows by taking the maximum of the above bounds.

In the following, we write $\|\cdot\|_{k, p, M}$ for the canonical $W^{k, p}(M)$ norm. The pointwise bound obtained in Proposition 5.1 yields the integral bound 1

$$
\begin{aligned}
& \Phi(R(\beta), \alpha)-\Phi(R(\alpha), \alpha)+\Phi(R(\alpha), \beta)-\Phi(R(\beta), \beta) \\
& \leq\left\|a \bar{\sigma}_{n} \max (|R(\alpha)|,|R(\beta)|) \log \left(V_{m}(\alpha) / V_{m}(\beta)\right)\right\|_{0,1, \Gamma_{F}} \\
&+\left\|a \bar{\sigma}_{n}\left[V_{m}(\alpha)-V_{m}(\beta)\right]\right\|_{0,1, \Gamma_{F}} \\
& \leq\|\max (|R(\alpha)|,|R(\beta)|)\|_{0,2, \Gamma_{F}}\left\|a \bar{\sigma}_{n} \log \left(V_{m}(\alpha) / V_{m}(\beta)\right)\right\|_{0,2, \Gamma_{F}} \\
&+\left\|a \bar{\sigma}_{n}\right\|_{0, \infty, \Gamma_{F}}\left\|V_{m}(\alpha)-V_{m}(\beta)\right\|_{0,1, \Gamma_{F}} \\
& \leq C_{1}\|\max (|R(\alpha)|,|R(\beta)|)\|_{0,2, \Gamma_{F}}\|\beta-\alpha\|_{0,2, \Gamma_{F}} \\
&+C_{2}\left\|e^{-(b / a) \alpha}-e^{-(b / a) \beta}\right\|_{0,1, \Gamma_{F}}
\end{aligned}
$$

with the constants $C_{1}:=\left\|b \bar{\sigma}_{n}\right\|_{0, \infty, \Gamma_{F}}$ and

$$
C_{2}:=\left\|a \bar{\sigma}_{n}\right\|_{0, \infty, \Gamma_{F}}\left\|V_{0} \exp \left(-\mu_{0} / a-(b / a) \log \left(V_{0} / L\right)\right)\right\|_{0, \infty, \Gamma_{F}},
$$

both of which are finite by assumptions (A.2) (A.7), In conjunction with (5.1), we thus have

$$
\begin{aligned}
C\|R(\alpha)-R(\beta)\|_{1,2, \Omega}^{2} \leq & C_{1}\|\max (|R(\alpha)|,|R(\beta)|)\|_{0,2, \Gamma_{F}}\|\beta-\alpha\|_{0,2, \Gamma_{F}} \\
& +C_{2}\left\|e^{-(b / a) \alpha}-e^{-(b / a) \beta}\right\|_{0,1, \Gamma_{F}} .
\end{aligned}
$$

On the one hand, $\alpha=S(V)$ and $\beta=0$ now turn $(5.3)$ into

$$
\begin{aligned}
& C\left(\|R S(V)\|_{1,2, \Omega}-\|R(0)\|_{1,2, \Omega}\right)^{2} \\
& \leq C\|R S(V)-R(0)\|_{1,2, \Omega}^{2} \\
& \leq C_{1}\|\max (|R S(V)|,|R(0)|)\|_{0,2, \Gamma_{F}}\|S(V)\|_{0,2, \Gamma_{F}} \\
&+C_{2}\left\|e^{-(b / a) S(V)}-1\right\|_{0,1, \Gamma_{F}} \\
& \leq C_{1}\left(\|R S(V)\|_{0,2, \Gamma_{F}}+\|R(0)\|_{0,2, \Gamma_{F}}\right)\|S(V)\|_{0,2, \Gamma_{F}} \\
&+C_{2}\left\|e^{-(b / a) S(V)}\right\|_{0,1, \Gamma_{F}}+C_{2}\|1\|_{0,1, \Gamma_{F}},
\end{aligned}
$$

which we can use to bound the growth of $R S$. On the other hand, once we know that $R S$ is bounded and show that $V_{n} \rightarrow V$ in $L^{2+\delta}\left(\Gamma_{F}\right)$ implies $S\left(V_{n}\right) \rightarrow S(V)$ in $L^{2}\left(\Gamma_{F}\right)$ and $e^{-(b / a) S\left(V_{n}\right)} \rightarrow e^{-(b / a) S(V)}$ in $L^{1}\left(\Gamma_{F}\right)$, it follows from 5.3 that $R S$ is continuous. To that end, we need to investigate $S$ more thoroughly.

5.2. An explicit formulation for the state problem. In this section it is shown that $S$ can also be viewed as a superposition operator.

We first observe that an application of the backward Euler scheme to Dieterich's law 2.15 turns it into

$$
\alpha_{n}-\tau e^{-\alpha_{n}}=\alpha_{n-1}-\frac{\Delta U_{n}}{L},
$$

with $\Delta U_{n}:=\tau V_{n}$. This prompts us to investigate the abstract problem

$$
z-\tau e^{-z}=r
$$

for which it is convenient to introduce the Lambert $W$ function [8].

Definition. For $z \in[0, \infty)$, we uniquely define $W(z)$ by $W(z) e^{W(z)}=z$.

\footnotetext{
${ }^{1}$ Here and in what follows, we do not distinguish between $v \in H$ and $\gamma_{F}(v) \in L^{p}\left(\Gamma_{F}\right)^{d}$ if the intended meaning is clear from the context.
} 
We can then write $z=W\left(\tau e^{-r}\right)+r$ for $(5.6)$ and

$$
\alpha_{n}=W\left(\tau e^{\Delta U_{n} / L-\alpha_{n-1}}\right)-\left(\Delta U_{n} / L-\alpha_{n-1}\right)
$$

for (5.5). If we furthermore define $s(V):=W\left(\tau e^{V}\right)-V$ and $s^{\alpha}(V):=s(V-\alpha)$, (5.5) takes the form

$$
\alpha_{n}=s^{\alpha_{n-1}}\left(\Delta U_{n} / L\right) .
$$

Proposition 5.2. The function $s$ is Lipschitz continuous and so is $s^{\alpha}$. As a consequence, $T_{s^{\alpha}}$ is a well-defined Lipschitz continuous operator from $L^{p}\left(\Gamma_{F}\right)$ to $L^{p}\left(\Gamma_{F}\right)$ whenever $\alpha \in L^{p}\left(\Gamma_{F}\right)$.

Proof. It is straightforward to show $W(z)^{\prime}=W(z) /[z(1+W(z))]$, which implies

$$
s^{\prime}(z)=\frac{W\left(\tau e^{z}\right)}{1+W\left(\tau e^{z}\right)}-1=-\frac{1}{1+W\left(\tau e^{z}\right)}
$$

and thus $\left|s^{\prime}\right|<1$ since we have $W(z)>0$ for $z>0$.

Given that Subproblem $\left(\mathrm{S}_{\tau}\right)$ with $\alpha_{n-1} \in L^{2}\left(\Gamma_{F}\right)$ has a unique solution over $L^{2}\left(\Gamma_{F}\right)$ by Proposition 4.4 and $T_{s^{\alpha}{ }_{n-1}}$ maps to $L^{2}\left(\Gamma_{F}\right)$ by Proposition 5.2 the two operators $S$ and $T_{s^{\alpha_{n-1}}}$ must coincide.

5.3. Growth of $S$. As a consequence of (5.4), the growth of $R S$ is dominated by the growth of $S$ and $e^{-(b / a) S}$. In this section, we show that $S$ has asymptotically logarithmic growth, so that we can shift our attention to $e^{-(b / a) S}$. To this end, we first show that $s$ has logarithmic growth on the positive real axis.

Lemma 5.3. We have $|s(z)| \leq \log (z / \tau)$ for $\tau \leq 1$ and $z \geq 1-\log \tau$.

Proof. Since $z \geq \tau$ implies $W\left(\tau e^{z}\right) \leq z$, we can assume $|s(z)|=-s(z)$. For $\tau=1$, we have

$$
y-W\left(e^{y}\right) \leq \log y \Longleftrightarrow(y-\log y) e^{y-\log y} \leq e^{y} \Longleftrightarrow y-\log y \leq y \Longleftrightarrow 1 \leq y .
$$

For the general case, with $\tau \leq 1$ and $z+\log \tau \geq 1$ the above implies

$$
-s(z)+\log \tau=(z+\log \tau)-W\left(\tau e^{z}\right) \leq \log (z+\log \tau) \leq \log z
$$

from which the claim immediately follows.

The operator $S$ inherits this property for non-negative arguments from $s$. To show this, we can use Jensen's inequality. The application is not straightforward, however, since $S$ is parametrised with a state $\alpha$, and $s^{2}$ is not concave on all of $\mathbb{R}$. We first address the second concern.

Lemma 5.4. Let $\tau>0$ be arbitrary. Then there is a $z_{0}(\tau) \geq 0$ such that

$$
\frac{d}{d z}\left(s(z)^{2}\right) \geq 0 \quad \text { and } \quad \frac{d^{2}}{d z^{2}}\left(s(z)^{2}\right) \leq 0
$$

for $z \geq z_{0}(\tau)$.

Proof. We find

$$
\frac{d}{d z}\left(s(z)^{2}\right)=2 \cdot \frac{z-W\left(\tau e^{z}\right)}{1+W\left(\tau e^{z}\right)} \geq 0 \Longleftrightarrow z \geq W\left(\tau e^{z}\right) \Longleftrightarrow z \geq \tau,
$$

so that we can choose any $z_{0} \geq \tau$ to make $s^{2}$ non-decreasing from $z_{0}$ on. We also find

$$
\begin{aligned}
\frac{d^{2}}{d z^{2}}\left(s(z)^{2}\right)=2 \cdot \frac{1+W\left(\tau e^{z}\right)+\left(W\left(\tau e^{z}\right)-z\right) W\left(\tau e^{z}\right)}{\left(1+W\left(\tau e^{z}\right)\right)^{3}} & \leq 0 \\
\Longleftrightarrow \frac{1+W\left(\tau e^{z}\right)}{W\left(\tau e^{z}\right)} & \leq z-W\left(\tau e^{z}\right),
\end{aligned}
$$


which must be true from some $z_{0} \geq 0$ on since the left-hand side of (5.7) converges to 1 from above and the right-hand side goes to infinity with $z \rightarrow \infty$.

Since now $s^{2}$ is concave and non-decreasing on an interval $\left[z_{0}, \infty\right)$, we can bound integrals over $s(z)^{2}$ with $z \geq z_{0}$.

Lemma 5.5. Assume $\tau \leq 1$. Then there is a constant $C$ such that for any $V \in$ $L^{p}\left(\Gamma_{F}\right)$ and $\alpha \in L^{2}\left(\Gamma_{F}\right)$ with $Z(V, \alpha):=\|V\|_{0, p, \Gamma_{F}}+\|\alpha\|_{0,2, \Gamma_{F}} \geq C$ and $M:=\{x \in$ $\left.\Gamma_{F}: V(x)-\alpha(x) \geq z_{0}\right\}$, we have

$$
\left(\int_{M} s(V-\alpha)^{2}\right)^{1 / 2} \leq \lambda\left(\Gamma_{F}\right)^{1 / 2} \log \left(\frac{C\left(\Gamma_{F}\right)}{\tau} Z(V, \alpha)\right)+2 / e,
$$

where $\lambda$ denotes the $(d-1)$-dimensional Lebesgue measure.

Proof. Since the case $\lambda(M)=0$ is trivially covered by choosing $C \geq \tau / C\left(\Gamma_{F}\right)$, we assume $\lambda(M)>0$ and define a function $g$ by

$$
g(z):= \begin{cases}s(z)^{2} & \text { if } z \geq z_{0} \\ s\left(z_{0}\right)^{2}+\left.\left(z-z_{0}\right) \frac{d}{d z}\left(s(z)^{2}\right)\right|_{z=z_{0}} & \text { otherwise }\end{cases}
$$

so that $g$ is non-decreasing, concave, and coincides with $s^{2}$ on $\left[z_{0}, \infty\right)$. By construction, we now have

$$
\begin{aligned}
\frac{1}{\lambda(M)} \int_{M} s(V-\alpha)^{2} & =\int_{M} g(V-\alpha) \frac{d \lambda}{\lambda(M)} \\
& \leq g\left(\int_{M} V-\alpha \frac{d \lambda}{\lambda(M)}\right) \\
& \leq g\left(\frac{C\left(\Gamma_{F}\right)}{\lambda(M)} Z(V, \alpha)\right) \\
& =s\left(\frac{C\left(\Gamma_{F}\right)}{\lambda(M)} Z(V, \alpha)\right)^{2} \quad \text { if } \frac{C\left(\Gamma_{F}\right)}{\lambda\left(\Gamma_{F}\right)} Z(V, \alpha) \geq z_{0} \\
& \leq \log \left(\frac{C\left(\Gamma_{F}\right)}{\tau \lambda(M)} Z(V, \alpha)\right)^{2} \quad \text { if } \frac{C\left(\Gamma_{F}\right)}{\lambda\left(\Gamma_{F}\right)} Z(V, \alpha) \geq 1-\log \tau
\end{aligned}
$$

by Jensen's inequality, Lemma 5.3 and Lemma 5.4 . This means

$$
\left(\int_{M} s(V-\alpha)^{2}\right)^{1 / 2} \leq \lambda(M)^{1 / 2} \log \left(\frac{C\left(\Gamma_{F}\right)}{\tau \lambda(M)} Z(V, \alpha)\right)
$$

whenever $Z(V, \alpha) \geq \tilde{C}:=\max \left\{z_{0}, 1-\log \tau\right\} \lambda\left(\Gamma_{F}\right) / C\left(\Gamma_{F}\right)$. We conclude

$$
\begin{aligned}
\left(\int_{M} s(V-\alpha)^{2}\right)^{1 / 2} & \leq \lambda(M)^{1 / 2} \log \left(\frac{C\left(\Gamma_{F}\right)}{\tau} Z(V, \alpha)\right)-\lambda(M)^{1 / 2} \log (\lambda(M)) \\
& \leq \lambda\left(\Gamma_{F}\right)^{1 / 2} \log \left(\frac{C\left(\Gamma_{F}\right)}{\tau} Z(V, \alpha)\right)+2 / e
\end{aligned}
$$

whenever $Z(V, \alpha) \geq \max \left\{\tilde{C}, \tau / C\left(\Gamma_{F}\right)\right\}$.

To show that $S$ has asymptotically logarithmic growth, we now only need to make sure that points $x$ with $V(x)<z_{0}$ can be neglected.

Lemma 5.6. We have $s(z)^{2} \leq z^{2}+1 / \tau$ for all $z$ and $\tau \leq 1$.

Proof. The substitution $z=y / \tau+\log \left(y / \tau^{2}\right)$ reduces the claim to

$$
2 \frac{y}{\tau} \log \left(\tau^{2}\right)-\frac{y^{2}}{\tau^{2}} \leq 2 \frac{y}{\tau} \log y+\frac{1}{\tau}
$$


with $y>0$. Since the left-hand side of $(5.8)$ is negative, this is implied by

$$
0 \leq 2 y \log y+1
$$

which is obvious.

Combining the above observations yields the result.

Proposition 5.7. Assuming $\tau \leq 1$ and $\alpha \in L^{p}\left(\Gamma_{F}\right)$, we have $\left\|s^{\alpha}(V)\right\|_{0,2, \Gamma_{F}} \in$ $O\left(\log \|v\|_{0, p, \Gamma_{F}}\right)$ for $\|V\|_{0, p, \Gamma_{F}} \rightarrow \infty$.

Proof. We first observe

$$
\left\|s^{\alpha}(V)\right\|_{0,2} \leq\left(\int_{V-\alpha<z_{0}} s(V-\alpha)^{2}\right)^{1 / 2}+\left(\int_{V-\alpha \geq z_{0}} s(V-\alpha)^{2}\right)^{1 / 2}
$$

and

$$
\begin{aligned}
\left(\int_{V-\alpha<z_{0}} s(V-\alpha)^{2}\right)^{1 / 2} & \leq\left(\int_{V-\alpha<z_{0}}(V-\alpha)^{2}+1 / \tau\right)^{1 / 2} \\
& \leq\left(\int_{V-\alpha<0} \alpha^{2}+1 / \tau\right)^{1 / 2}+\left(\int_{0 \leq V-\alpha<z_{0}} z_{0}^{2}+1 / \tau\right)^{1 / 2} \\
& \leq\|\alpha\|_{0,2, \Gamma_{F}}+2\|1 / \tau\|_{0,1, \Gamma_{F}}^{1 / 2}+\left\|z_{0}\right\|_{0,2, \Gamma_{F}}
\end{aligned}
$$

with arbitrary $V \in L^{p}\left(\Gamma_{F}\right)$ by virtue of Lemma 5.6. Now choose $C$ in accordance with Lemma 5.5. Either we have $\|V\|_{0, p, \Gamma_{F}}+\|\alpha\|_{0,2, \Gamma_{F}} \geq C$, so that

$$
\left(\int_{V-\alpha \geq z_{0}} s(V-\alpha)^{2}\right)^{1 / 2} \leq \lambda\left(\Gamma_{F}\right)^{1 / 2} \log \left(\frac{C\left(\Gamma_{F}\right)}{\tau}\left[\|V\|_{0, p, \Gamma_{F}}+\|\alpha\|_{0,2, \Gamma_{F}}\right]\right)+2 / e,
$$

which means

$$
\left\|s^{\alpha}(V)\right\|_{0,2, \Gamma_{F}} \in O\left(\log \|V\|_{0, p, \Gamma_{F}}\right) \quad \text { with }\|V\|_{0, p, \Gamma_{F}} \rightarrow \infty
$$

as claimed, or $\|V-\alpha\|_{0,2, \Gamma_{F}}$ is bounded by a constant and so is $\|s(V-\alpha)\|_{0,2, \Gamma_{F}}$ by Proposition 5.2 , so that the claim is trivially true.

Remark. In the same manner, we obtain $\left\|s^{\alpha}(|v|)\right\|_{0,2, \Gamma_{F}} \in O\left(\log \|v\|_{1,2, \Omega}\right)$ with $\|v\|_{1,2, \Omega} \rightarrow \infty$.

5.4. Growth of $e^{-(b / a) S}$. We now bound the growth of $e^{-S}:=T_{\exp \left(-s^{\alpha_{n}-1}\right)}$; again by investigating the underlying scalar map.

Proposition 5.8. The function $e^{-s}$ is Lipschitz continuous.

Proof. We have

$$
\left|\frac{d}{d z} e^{-s(z)}\right|=\frac{e^{z-W\left(\tau e^{z}\right)}}{1+W\left(\tau e^{z}\right)} \leq 1 / \tau
$$

since

$$
\tau e^{z-W\left(\tau e^{z}\right)}=W\left(\tau e^{z}\right) \leq 1+W\left(\tau e^{z}\right) .
$$

As a consequence, the operator $e^{-S}$ is obviously well-defined and Lipschitz continuous from $L^{p}\left(\Gamma_{F}\right)$ to $L^{p}\left(\Gamma_{F}\right)$ whenever $\alpha_{n-1} \in L^{p}\left(\Gamma_{F}\right)$. We also have

$$
\left\|e^{-r S(V)}\right\|_{0,1, \Gamma_{F}}=\left\|e^{-S(V)}\right\|_{0, r, \Gamma_{F}}^{r} \in O\left(\|V\|_{0, r, \Gamma_{F}}^{r}\right)
$$

for any $r \leq p$.

Corollary 5.9. From $\alpha_{n-1}, e^{-\alpha_{n-1}} \in L^{p}\left(\Gamma_{F}\right)$ and $u \in L^{p}\left(\Gamma_{F}\right)^{d}$ it follows that $\alpha_{n}$, $e^{-\alpha_{n}} \in L^{p}\left(\Gamma_{F}\right)$. In other words, regularity of the state variable is carried over from one time step to the next.

Proof. This is an immediate consequence of Propositions 5.2 and 5.8 
It should be noted that the analogue of Corollary 5.9 for Ruina's law does not seem to hold.

5.5. Growth and continuity of $R S$. With Proposition 5.7 and Proposition 5.8 we have bounded the growth of the right-hand side of $(5.4)$. In this section, we collect the implications for the operator $R S$. We first need a technical lemma.

Lemma 5.10. From $f(t)^{2} \in O\left(f(t) \log t+t^{r}\right)$ with $r>0$ it follows that

$$
f(t) \in O\left(t^{r / 2}\right) \text { with } t \rightarrow \infty \text {. }
$$

Proof. Straightforward.

Proposition 5.11. Assume $b / a \leq r$ with $r \leq 2$. We then have

$$
\|R S(V)\|_{1,2, \Omega} \in O\left(\|V\|_{0, r, \Gamma_{F}}^{r / 2}\right)
$$

for $\|V\|_{0, r, \Gamma_{F}} \rightarrow \infty$. In particular, $R S$ is then bounded.

Proof. From (5.4) and (5.9) as well as Proposition 5.7 we deduce

$$
\begin{aligned}
\|R S(V)\|_{1,2, \Omega}^{2} & \in O\left(\|R S(V)\|_{1,2, \Omega}\|S(V)\|_{0,2, \Gamma_{F}}+\left\|e^{-(b / a) S(V)}\right\|_{0,1, \Gamma_{F}}\right) \\
& \subseteq O\left(\|R S(V)\|_{1,2, \Omega} \log \|V\|_{0, r, \Gamma_{F}}+\|V\|_{0, r, \Gamma_{F}}^{r}\right) .
\end{aligned}
$$

The claim now follows from Lemma 5.10

Remark. In a similar fashion, we conclude

$$
\|R S(|u|)\|_{1,2, \Omega} \in O\left(\|u\|_{1,2, \Omega}^{r / 2}\right)
$$

for $b / a \leq r<p^{*}$.

Corollary 5.12. If we, furthermore, assume $r<2$, we obtain

$$
\|R S(|u|)\|_{1,2, \Omega} \in o\left(\|u\|_{1,2, \Omega}\right),
$$

so that $R S \circ\left|\gamma_{F}\right|$ is a self-map on sufficiently large balls in $H$.

The assumption $b / a \leq r<2$ is not unreasonable. The literature has this to say:

"Laboratory experiments generally show $a \approx 2(b-a)$ " [26].

which implies $b / a \approx 3 / 2$. From another source:

"Laboratory values of $a / b$ are typically larger than $0.5 "$ " [1].

In order to apply Schauder's theorem, we now only need to show that $R S$ is continuous.

Proposition 5.13. The operator $R S: L^{p}\left(\Gamma_{F}\right) \rightarrow H$ with $p \geq 2$ is continuous whenever $b / a \leq p$ and $\alpha_{n-1} \in L^{p}\left(\Gamma_{F}\right)$.

Proof. Let $V_{n}$ converge to $V \in L^{p}\left(\Gamma_{F}\right)$. We know that the sequence $R S\left(V_{n}\right)$ is bounded from Proposition 5.11, that $S\left(V_{n}\right)$ converges in $L^{2}$ from Proposition 5.2 and that $e^{-(b / a) S(V)}$ converges in $L^{1}$ from Proposition 5.8. In summary, the righthand side of (5.3) converges and so must the left-hand side.

We thus have that for $b / a \leq r<2$, the operator $R S \circ\left|\gamma_{F}\right|$ is a continuous compact self-map on large balls in $H$. By Schauder's fixed point theorem [12, Corollary 11.2], such balls contain fixed points of $R S \circ\left|\gamma_{F}\right|$. We conclude:

Theorem 5.14. If we have $\|b / a\|_{0, \infty, \Gamma_{F}} \leq r<2$ as well as $\alpha_{n-1} \in L^{2}\left(\Gamma_{F}\right)$ and $e^{-\alpha_{n-1}} \in L^{2}\left(\Gamma_{F}\right)$, then the time-discrete coupled problem corresponding to the $n^{\text {th }}$ time step with Dieterich's state evolution law has a solution. The new state satisfies $\alpha_{n} \in L^{2}\left(\Gamma_{F}\right)$ and $e^{-\alpha_{n}} \in L^{2}\left(\Gamma_{F}\right)$. 
Corollary 5.15. If we have $\|b / a\|_{0, \infty, \Gamma_{F}} \leq r<2$ as well as $\alpha_{0} \in L^{2}\left(\Gamma_{F}\right)$ and $e^{-\alpha_{0}} \in L^{2}\left(\Gamma_{F}\right)$, then the time-discrete coupled problem with Dieterich's state evolution law has a solution for every time step.

\section{Numerical Experiments}

6.1. A model problem. We consider a two-dimensional $5 \mathrm{~m} \times 1 \mathrm{~m}$ slider $\Omega$ as depicted in Figure 2.1 and assume it to consist of a St. Venant-Kirchhoff material. The body force shall represent gravity, hence we set $f=-\rho g \cdot e_{2}$. Here and in the following $e_{1}, e_{2}$ denote the unit vectors in $\mathbb{R}^{2}$.

The top of the slider moves with a constant velocity of $2 \times 10^{-4} \mathrm{~m} / \mathrm{s}$ in direction of $e_{1}$, which we prescribe through a Dirichlet boundary condition on $\Gamma_{D}$. The bottom $\Gamma_{F}$ obeys a Dieterich-Ruina friction model, and on $\Gamma_{N}$ homogeneous Neumann conditions $f_{N}=0$ are imposed.

We select homogeneous initial data $u(\cdot, 0)=\dot{u}(\cdot, 0)=0$ on $\Omega$, and the initial condition $\alpha(\cdot, 0)=\alpha_{0}$ with $\alpha_{0}=e^{-10}$ on $\Gamma_{F}$. The remaining parameters are listed in Table 6.1

We select the final time $T=15 \mathrm{~s}$ and the time step $\tau=T / N$ with $N=10^{4}$, if not stated otherwise. Spatial discretisation of Subproblem $\left(\mathbf{R}_{\tau}\right)$, i.e., of the velocity $\dot{u}_{n}$, is carried out with respect to a triangular grid $\mathcal{T}_{j}$ arising from $j$ successive uniform refinements of a regular initial grid $\mathcal{T}_{0}$ with $2 \times 6$ vertices and corresponding piecewise linear finite elements $\mathcal{S}_{j} \subset H$. For the states $\alpha_{n}, n=1, \ldots, N$, we use piecewise constant finite elements on the dual of the trace grid $\mathcal{T}_{j} \cap \Gamma_{F}$. The implementation is based on the DunE libraries [2].

6.2. Convergence properties of the fixed point iteration. In light of the theoretical considerations in Section 4. we first investigate the convergence of the fixed point iteration

$$
\dot{u}_{n, j}^{\nu+1}=R_{j}\left(\omega^{\nu} \alpha_{n, j}^{\nu+1}+\left(1-\omega^{\nu}\right) \alpha_{n, j}^{\nu}\right), \quad \alpha_{n, j}^{\nu+1}=S_{j}\left(\left|\gamma_{F}\left(\dot{u}_{n, j}^{\nu}\right)\right|\right), \quad \nu=0,1, \ldots
$$

for the algebraic spatial problems (see Section 6.1) numerically. We select the damping parameter $\omega^{\nu}=1$, if $\nu=0,1$ or

$$
\left\|\alpha_{n, j}^{\nu+1}-\alpha_{n, j}^{\nu}\right\|_{L^{2}\left(\Gamma_{F}\right)} \leq \frac{1}{2}\left\|\alpha_{n, j}^{\nu}-\alpha_{n, j}^{\nu-1}\right\|_{L^{2}\left(\Gamma_{F}\right)}, \quad \nu=2,3, \ldots,
$$

and $\omega^{\nu}=0.5$ otherwise.

The evaluation of $S_{j}$, i.e., the solution of a discrete version of $\left(\mathrm{S}_{\tau}\right)$ is computed pointwise by a bisection method up to a pointwise absolute error of $10^{-12}$.

The evaluation of the discrete operator $R_{j}$, i.e., the solution of the discrete counterpart of the smooth, convex minimisation problem $\left(\mathrm{R}_{\tau}\right)$ on the finite-dimensional space $\mathcal{S}_{j} \subset H$ is performed iteratively by Truncated Nonsmooth Newton Multigrid iterations (TNNMG) [14, 15, 16] with an absolute error tolerance of $10^{-10}$ with respect to the norm $\|\cdot\|$ given by

$$
\|v\|=\left((\mathcal{C} \varepsilon(v), \varepsilon(v))_{L^{2}(\Omega)}+(\rho v, v)_{L^{2}(\Omega)}\right)^{1 / 2}, \quad v \in H .
$$

\begin{tabular}{l|c||l|c} 
Parameter & Value & Parameter & Value \\
\hline Mass density $\rho$ & $5 \times 10^{3} \mathrm{~kg} / \mathrm{m}^{2}$ & Ref. velocity $V_{0}$ & $1 \times 10^{-6} \mathrm{~m} / \mathrm{s}$ \\
Poisson's ratio $\nu$ & 0.3 & Ref. friction coeff. $\mu_{0}$ & 0.6 \\
Young's modulus $E$ & $5 \times 10^{7} \mathrm{~N} / \mathrm{m}$ & Rate-effect coeff. $a$ & 0.010 \\
Gravity $g$ & $9.81 \mathrm{~N} / \mathrm{kg}$ & State-effect coeff. $b$ & 0.015 \\
Prescr. normal stress $\bar{\sigma}_{n}$ & $49050 \mathrm{~N} / \mathrm{m}$ & Characteristic slip dist. $L$ & $1 \times 10^{-5} \mathrm{~m}$
\end{tabular}

TABLE 6.1. Material parameters 
The overall fixed point iteration is stopped once the criterion

$$
\left\|u_{n, j}^{\nu+1}-u_{n, j}^{\nu}\right\| \leq 10^{-10}
$$

is satisfied.

Both for Dieterich's law (left) and Ruina's law (right), Figure 6.1 shows the average (solid) and maximum number (dashed) of required fixed point iterations per time step for the spatial problems arising in $10^{4}$ time steps over the number of refinement levels $j$ of the underlying triangulation $\mathcal{T}_{j}, j=2, \ldots, 8$. In both cases, the number of required fixed point iterations appears to saturate with decreasing mesh size, suggesting mesh-independent convergence. A theoretical justification is the subject of future research.
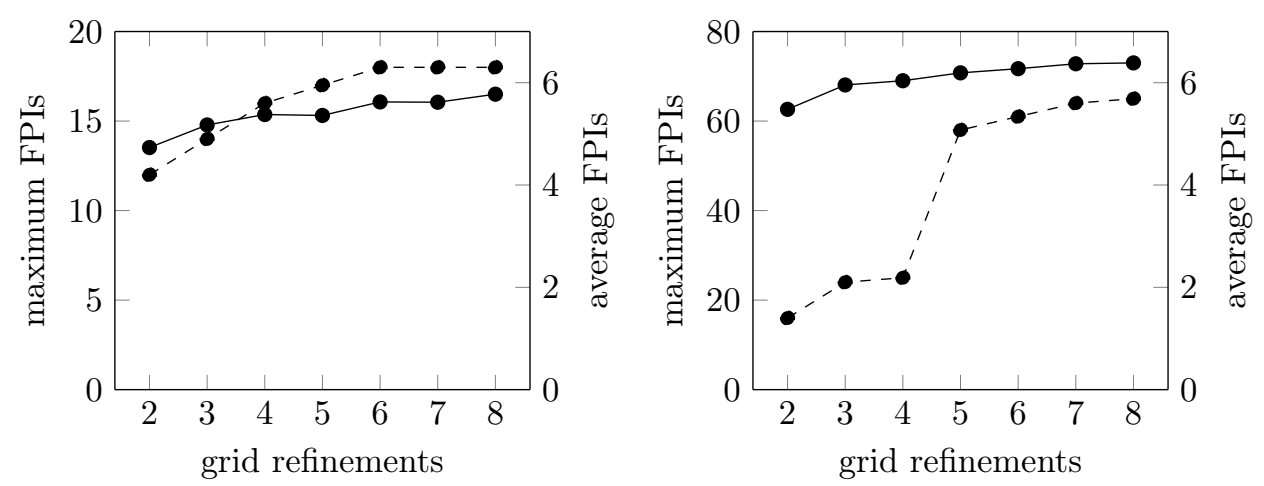

Figure 6.1. Average (solid) and maximum (dashed) number of fixed point iterations per time step required for $10^{4}$ time steps over the number of refinement levels $j$ for Dieterich's law (left) and Ruina's law (right).

6.3. Convergence properties of the discretisation. During the time interval $[0, T]$, the evolution of the body $\Omega$ goes through three phases: It first shears without slipping (roughly for $t_{n} \in[0,0.4 T]$ ), then it starts to slip unsteadily (roughly for $\left.t_{n} \in[0.4 T, 0.75 T]\right)$, and finally - both for Dieterich's and Ruina's law - it enters a regime of quasiperiodic slip events.

We concentrate on Dieterich's law and the quasiperiodic regime. To get an idea of the total movement of the object we examine the displacement $\gamma_{F}\left(u_{j}\right)\left(x_{0}, \cdot\right)$ and sliding velocity $\left|\gamma_{F}\left(\dot{u}_{j}\right)\left(x_{0}, \cdot\right)\right|$ at the centre $x_{0}$ of $\Gamma_{F}$. Discretisation is carried out using the Newmark/finite element methodology described in Sections 3 and 6.1. The triangulation $\mathcal{T}_{j}, j=4$ is fixed and we consider a sequence of decreasing time step sizes $\tau_{i}=T / N_{i}, N_{i}=10^{4+i}, i=0,1,2$. The resulting approximate displacements and velocities are shown in Figures 6.2 and 6.3 respectively. Note the quasiperiodic steps (displacement) and peaks (velocity) associated with slip events. Each of these periods is resolved by about $10^{3}-10^{5}$ time steps. For the step sizes $\tau_{1}$ and $\tau_{2}$, we obtain strong agreement of both approximate displacement and velocity. Note that frequency and amplitude of the oscillations displayed by the sliding velocity appear to increase with decreasing time step size $\tau$.

We found the same qualitative behaviour for Ruina's law. However, grid convergence seems to require even smaller values of $\tau$ that might be prohibitive in practical calculations. More efficient discretisation schemes are the subject of current research. 


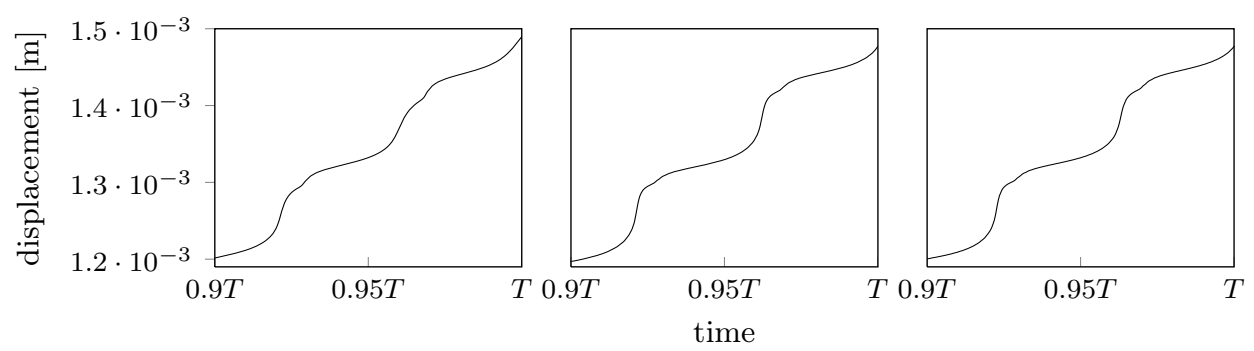

Figure 6.2. Dieterich's law: Displacement at the centre $x_{0}$ of the frictional boundary $\Gamma_{F}$ for the time step sizes $\tau_{i}=T / 10^{4+i}, i=$ $0,1,2$ (left to right).

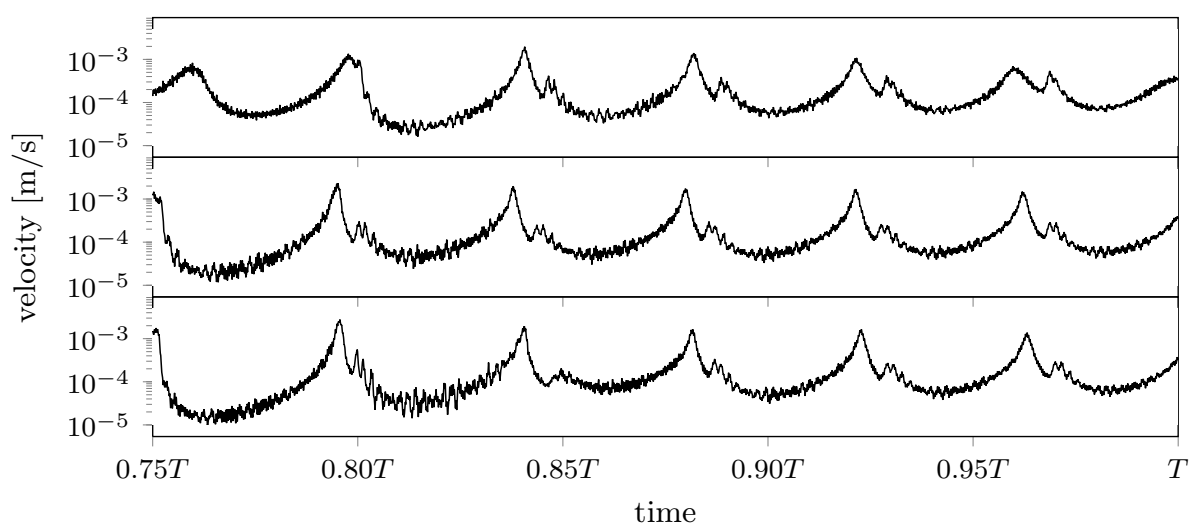

FiguRE 6.3. Dieterich's law: Velocity at the centre $x_{0}$ of the frictional boundary $\Gamma_{F}$ for the time step sizes $\tau_{i}=T / 10^{4+i}, i=0,1,2$ (top to bottom).

\section{REFERENCES}

1. Jean-Paul Ampuero and Allan M. Rubin, Earthquake nucleation on rate and state faults aging and slip laws, J. Geophys. Res. 113 (2008), no. B1, B01302.

2. P. Bastian, M. Blatt, A. Dedner, C. Engwer, R. Klöfkorn, R. Kornhuber, M. Ohlberger, and O. Sander, A generic interface for adaptive and parallel scientific computing. Part II: Implementation and tests in DUNE, Computing 82 (2008), no. 2-3, 121-138.

3. N. M. Beeler, T. E. Tullis, and J. D. Weeks, The roles of time and displacement in the evolution effect in rock friction, Geophys. Res. Lett. 21 (1994), no. 18, 1987-1990.

4. Yehuda Ben-Zion, Collective behavior of earthquakes and faults: Continuum-discrete transitions, progressive evolutionary changes, and different dynamic regimes, Rev. Geophys. 46 (2008), no. 4, RG4006.

5. Yehuda Ben-Zion and James R. Rice, Dynamic simulations of slip on a smooth fault in an elastic solid, J. Geophys. Res. 102 (1997), no. B8, 17771-17784.

6. Markus Biegert, On traces of Sobolev functions on the boundary of extension domains, Proc. Amer. Math. Soc. 137 (2009), no. 12, 4169-4176.

7. Ronaldo I. Borja and Craig D. Foster, Continuum mathematical modeling of slip weakening in geological systems, J. Geophys. Res. 112 (2007), no. B4, B04301.

8. R. Corless, G. Gonnet, D. Hare, D. Jeffrey, and D. Knuth, On the Lambert $W$ function, Advances in Computational Mathematics 5 (1996), 329-359.

9. Christof Eck, Jiří Jarušek, and Miroslav Krbec, Unilateral contact problems: Variational methods and existence theorems, Pure and Applied Mathematics (Boca Raton), vol. 270, Chapman \& Hall/CRC, Boca Raton, FL, 2005.

10. Åke Fagereng and Virginia G. Toy, Geology of the earthquake source: an introduction, Geological Society, London, Special Publications 359 (2011), no. 1, 1-16. 
11. Irene Fonseca and Giovanni Leoni, Modern methods in the calculus of variations: $L^{p}$ spaces, Springer Monographs in Mathematics, Springer, New York, 2007.

12. David Gilbarg and Neil S. Trudinger, Elliptic partial differential equations of second order, Springer-Verlag, Berlin, 1977, Grundlehren der Mathematischen Wissenschaften, Vol. 224.

13. Roland Glowinski, Numerical methods for nonlinear variational problems, Springer Series in Computational Physics, Springer-Verlag, New York, 1984

14. C. Gräser, Convex minimization and phase field models, Ph.D. thesis, Freie Universität Berlin, 2011.

15. C. Gräser and R. Kornhuber, Multigrid methods for obstacle problems, J. Comp. Math. 27 (2009), no. 1, 1-44.

16. Carsten Gräser, Uli Sack, and Oliver Sander, Truncated nonsmooth Newton multigrid methods for convex minimization problems, Domain Decomposition Methods in Science and Engineering XVIII (Timothy J. Barth, Michael Griebel, David E. Keyes, Risto M. Nieminen, Dirk Roose, and Tamar Schlick, eds.), Lecture Notes in Computational Science and Engineering, vol. 70, Springer Berlin Heidelberg, 2009, pp. 129-136.

17. Ji-Cheng Gu, James R. Rice, Andy L. Ruina, and Simon T. Tse, Slip motion and stability of a single degree of freedom elastic system with rate and state dependent friction, Journal of the Mechanics and Physics of Solids 32 (1984), no. 3, 167-196.

18. N. Kikuchi and J. T. Oden, Contact problems in elasticity: a study of variational inequalities and finite element methods, SIAM Studies in Applied Mathematics, vol. 8, Society for Industrial and Applied Mathematics (SIAM), Philadelphia, PA, 1988.

19. Chris Marone, Laboratory-derived friction laws and their application to seismic faulting, Annual Review of Earth and Planetary Sciences 26 (1998), no. 1, 643-696.

20. V.L. Popov, B. Grzemba, J. Starcevic, and C. Fabry, Accelerated creep as a precursor of friction instability and earthquake prediction, Physical Mesomechanics 13 (2010), no. 5-6, $283-291$.

21. K. Ranjith and J. R. Rice, Stability of quasi-static slip in a single degree of freedom elastic system with rate and state dependent friction, Journal of the Mechanics and Physics of Solids 47 (1999), no. 6, 1207-1218.

22. J. R. Rice and Y. Ben-Zion, Slip complexity in earthquake fault models, Proceedings of the National Academy of Sciences 93 (1996), no. 9, 3811-3818.

23. J. R. Rice and A. L. Ruina, Stability of steady frictional slipping, J. Appl. Mech (1983), $343-349$.

24. James R. Rice, Nadia Lapusta, and K. Ranjith, Rate and state dependent friction and the stability of sliding between elastically deformable solids, Journal of the Mechanics and Physics of Solids 49 (2001), no. 9, 1865-1898.

25. R. Tyrrell Rockafellar, Convex analysis, Princeton Mathematical Series, No. 28, Princeton University Press, Princeton, N.J., 1970.

26. Mousumi Roy and Chris Marone, Earthquake nucleation on model faults with rate-and statedependent friction: Effects of inertia, J. Geophys. Res. 101 (1996), no. B6, 13919-13932.

27. Allan M. Rubin and Jean-Paul Ampuero, Self-similar slip pulses during rate-and-state earthquake nucleation, J. Geophys. Res. 114 (2009), no. B11, B11305.

28. Andy Ruina, Slip instability and state variable friction laws, J. Geophys. Res. 88 (1983), no. B12, 10359-10370.

29. Christopher H. Scholz, Earthquakes and friction laws, Nature 391 (1998), no. 6662, 37-42.

30. Alessandro Tiero, On Korn's inequality in the second case, Journal of Elasticity 54 (1999), $187-191$.

Elias Pipping, Freie Universität Berlin, Institut für Mathematik, Arnimallee 6, D 14195 Berlin, Germany

E-mail address: pipping@math.fu-berlin.de

Oliver Sander, RWTH Aachen University, Institut für Geometrie und Praktische Mathematik, Templergraben 55, D - 52062 Aachen, Germany

E-mail address: sander@igpm.rwth-aachen.de

Ralf Kornhuber, Freie Universität Berlin, Institut für Mathematik, Arnimallee 6 , D - 14195 Berlin, Germany

E-mail address: kornhuber@math.fu-berlin.de 